\title{
ENABLING SUSTAINABILITY AND ENERGY AWARENESS IN SCHOOLS BASED ON IOT AND REAL-WORLD DATA
}

\author{
Georgios Mylonas \\ CTI "Diophantus", Patras \\ Greece \\ Ioannis Chatzigiannakis \\ Sapienza University, Rome, \\ Italy \\ Dimitrios Amaxilatis \\ CTI "Diophantus", Patras \\ Greece \\ Federica Paganelli \\ CNIT, Florence, Italy \\ Aris Anagnostopoulos \\ Sapienza University, Rome, \\ Italy
}

Few loT systems monitoring energy consumption in buildings have focused on the educational community. loT in the educational domain can jumpstart a process of sustainability awareness and behavioral change towards energy savings, as well as provide tangible financial savings. We present a real-world multi-site loT deployment, comprising 19 school buildings, aiming at enabling loT-based energy awareness and sustainability lectures, promoting energy-saving behaviors supported by loT data. We discuss scenarios where loT-enabled applications are integrated into school life, providing an engaging and hands-on approach, based on real data, generating value in terms of educational and energy savings outcomes. We also present a set of first results, based on the analysis of school-building data, which highlight potential ways to identify irregularities and inefficiencies.

Raising awareness among young people and changing their habits concerning energy usage is considered key in achieving a sustainable energy behavior. A number of ongoing research projects utilize real-world IoT infrastructure to study the effects of behavioral change-based approaches to promote sustainability. However, although people under 30 represent a third of the EU's population [1], such IoT deployments typically focus on generic audiences, like people populating heterogeneous building fleets (e.g., a public/social housing buildings mix). Little part has focused exclusively on schools, students and teachers, thus leaving the investigation of IoT's potential impact in an educational setting largely unexplored.

The educational field seems ideal for utilizing IoT for monitoring and management systems for buildings. For instance, educational organizations operate thousands of buildings, while educational institutions revolve around students, educators, and policy makers, all essential in implementing energy-awareness policies. Furthermore, EU considers environmental education as one of the prominent instruments influencing human behavior towards environmental sustainability [2] and educational buildings constitute 17\% of EU's non-residential building stock (in $\mathrm{m}^{2}$ ) [3]. Moreover, evidence shows that a focus on energy use in schools yields multiple benefits, along with educational excellence and a healthy learning environment [4]. 
Having these in mind, we discuss an education-focused IoT platform comprising a real-world IoT infrastructure deployed in a number of educational buildings in Europe and a Service Platform offering uniform APIs for data access, supplemented by a software toolset aiming at both helping in educating students on energy and environmental matters, and at achieving better energy efficiency. This deployment has been developed in the context of the GAIA (Green Awareness In Action, gaia-project.eu) EU project. Our platform is currently operating in 19 school buildings in Greece, Italy and Sweden.

Leveraging this experience, we discuss how IoT technologies can be applied inside schools, creating educational value on the one hand, and on the other one financial benefits for schools. We expect to achieve multiple benefits by focusing on increasing energy awareness and behavioral change, and we provide some first results that point to such aspects, along with preliminary enduser feedback from trials inside schools.

\section{Previous Work}

The EU actively promotes energy efficiency initiatives like BuildUp (http://www.buildup.eu), a portal for gathering resources for energy efficiency in buildings. SMARTSPACES

(http://www.smartspaces.eu) utilized 11 pilot sites, researching energy efficiency in public buildings. More focused on school buildings are projects like ZEMEDS (http://www.zemeds.eu), producing recommendation and optimization components. Froehlich et al. [5] discusses common approaches in feedback design in eco-conscious work. School of the Future (http://www.schoolof-the-future.eu) produced several guidelines and results regarding good energy saving practices in an educational setting. Alvarez-Campana et al. [6] present a large-scale campus deployment whose main goal is to enable experimenting with Smart City services. SEACS [7] has produced a vast set of educational plans for energy efficiency, which can be realized over a deployment such as GAIA's. Our work aims to deliver a replicable solution, i.e., open-source and standardsbased wherever possible, that provides reliable real-time data to be used both as input for lectures and a means to provide financial savings. At the same time, it does so while being one of the largest and more distributed IoT infrastructures of its kind, to the best of our knowledge.

\section{EDUCATION-FOCUSED IOT AND ENERGY EFFICIENCY IN THE REAL WORLD}

A main objective of sustainability education and energy efficiency initiatives is making students aware that energy consumption is influenced by the sum of individual behaviors and that simple behavior changes and interventions have a tangible impact towards energy savings. To this end, IoT technologies can support such initiatives with immediate feedback regarding the impact of our actions and automating the implementation of energy-saving policies, while maintaining comfort. This allows on the one hand, better informing people and enabling them to make informed decisions, and on the other to enable a different set of applications, like gamification bridging the virtual world with the real one. The availability of actual measurements of parameters such as energy consumption, luminosity, temperature, etc., enables a number of diverse education-related applications and scenarios:

Education: Teachers use collected data and analytics during class to explain phenomena @ 20XX IEEE. Personal use of this material is permitted. Permission from IEEE must be obtained for all other uses, in any current or future media, including reprinting/republishing this material for advertising or promotional purposes, creating new collective works, for resale or redistribution to servers or lists, or reuse of any copyrighted component of this work in other works.

- related to parameters monitored.

- Student engagement: Teachers organize projects where students/groups monitor environmental parameters in class/home.

- End-user programming: Students develop software using data provided by the platform utilizing available APIs. 
- Building management: Collected data feed applications informing managers about their building's performance. Data from other schools supports decision-making.

- Scientific research: Data collected is made available to the scientific community, using common datasets and comparing results.

GAIA's IoT deployment is multi-site and multi-country. The level of abstraction introduced by an IoT service architecture on top of sensors by different manufacturers, together with the capability of performing data analytics and interpreting data with context information, widen the application scope of IoT. Monitoring school buildings situated in different countries can motivate, for example, to identify energy consumption patterns in such countries. This can be used to make comparisons/competitions; for instance, school A could compete with school B in efficiency.

This could also help in understanding cultural differences regarding energy awareness and sustainability.

\section{IREAL-WORLD SCHOOL PILOT DEPLOYMENT}

Our real-world deployment provides real-time monitoring of 19 school buildings spread in 3 countries (Greece, Italy and Sweden). The buildings are situated in different local climatic conditions: 3 buildings in suburban areas, 2 in rural areas, 2 in small islands, and the rest in city centers. The year of construction of these buildings ranges from 1950 to 2000 . We cover most educational levels (primary-high school) and different profiles (curricula, organization, building characteristics, regulations). The vast majority of the rooms monitored are used for teaching. In these schools, close to 1900 students and 370 teachers will be directly involved in GAIA.

The deployed devices provide 880 sensing points organized in four categories: (1) classroom environmental sensors; (2) atmospheric sensors (outdoors); (3) weather stations (on rooftops); and (4) power consumption meters (attached to main building electricity panels). Given the diverse building characteristics and usage requirements, deployments vary between schools (e.g., number of sensors, manufacturer, networking, etc.). The IoT devices used are either open-design IoT nodes (e.g., Arduino as described in GAIA's hardware design [8]), or off-the-shelf products from IoT device manufacturers. Figure 1 shows examples of the IoT devices used and their installation.

Indoor devices use IEEE 802.15.4 networks, forming ad hoc multi-hop networks. These devices are connected to cloud services via IoT gateway devices, which coordinate communication with the rest of the platform. Outdoor nodes use wired networking or WiFi. In some schools, multiple IoT gateways are required to cope with various issues (e.g., outdoor vs. indoor deployment across floors). The gateways communicate directly with our platform and support gateway-togateway communication, as well as device-to-device communication. The communication of the IoT devices with the monitoring infrastructure is done via various protocols and technologies, like MQTT components (http://mqtt.org), CoAP by Shelby et al [9], etc.

While this IoT deployment is being expanded to include additional buildings, we draw some lessons learnt from our experience. We had to deal with several issues, such as old buildings with thick walls having a very noticeable impact on the communication range of the devices used. The presence of large metal surfaces (e.g., doors), also had a tangible effect on networking inside buildings. Moreover, many schools have antiquated electricity installations, making it difficult to interface even with non-invasive IoT meters.

With respect to the stability of this IoT deployment, Figure 2 depicts the availability of measurements on a daily basis for each school separately. Schools are listed in chronological order of IoT deployment. In almost all deployments, there are values missing almost on a daily basis. These measurements are missing either because they were never reported to the cloud infrastructure, or due to a failure occurring during processing by the cloud services. In some cases, network failures occur either due to a packet transmission error at the network provider level (IEEE 802.15.4 or 802.11) or transmission errors between intermediate gateways and the cloud infrastructure over the Internet. The packet transmission errors were related to the buildings' layout, the location of the sensor devices within classrooms and the usage of the building. In some cases, (site A 
and C, Fig. 2) disconnections were addressed by installing additional gateway devices that improved wireless coverage. In other cases, (site D and F, Fig. 2) transmission errors were reduced by repositioning specific nodes to different locations within the same room. There are also cases where missing data were due to the users' actions: in one school, the students decided to disconnect some devices, while in another school, teachers decided to disconnect the complete network in an attempt to improve poor Internet connectivity. In such cases (site I, Fig. 2), we discussed the problem with the school staff and tried to improve end users' understanding of GAIA objectives by emphasizing the benefits of the project and the potential impact of sensors' data availability on educational activities. It is, therefore, crucial to coordinate with all involved people (i.e., teachers/students, building managers) to make sure that the platform's operation/continuity is maintained.

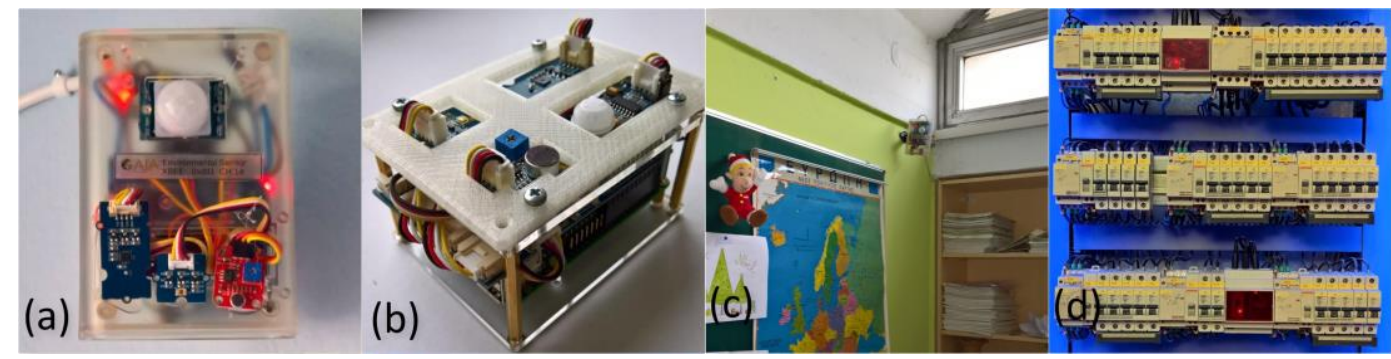

Fig. 1. Hardware nodes used and installations inside schools: an Arduino-based node (a), a Raspberry Pi-based (b), placement of nodes inside a classroom (c) and inside an electricity panel (d).

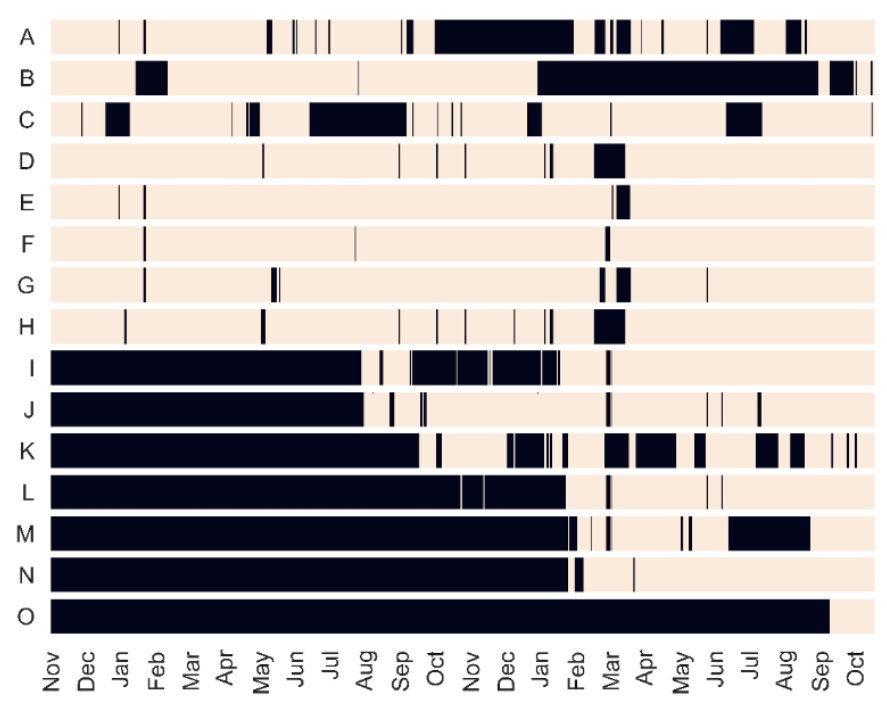

Fig. 2. Data availability per school building (11/2016-10/2017, for issues related to confidentiality the schools' names are omitted). A single dark point signifies missing data for the specific school on a specific day, while a white part signifies complete availability 


\title{
I IOT SERVICE PLATFORM
}

\author{
Main requirements and key design choices
}

Designing an IoT service platform targeting education entails a broad range of functional and non-functional requirements. Our platform is deployed over buildings with different characteristics and requirements (multi-site deployments). Devices from multiple providers work under the same interoperability framework (device heterogeneity). Different sensors are installed, from energy sensors to indoor and outdoor environmental sensors, motion sensors, etc., generating data uniformly accessible through a common set of APIs (data variety). The platform acts as an intermediation layer between deployments and a variety of applications that facilitate the educational sector towards improving the energy efficiency of school buildings (user diversity). The platform provides open and easy-to-use API interfaces that support the development of applications targeting different end-user groups that inherently exist in education (open design). Certain users (building managers) require real-time building monitoring, while others (students, teachers) require aggregated numbers for educational material and student projects (data velocity). In some cases, different school buildings are unified into a single view to make interaction simpler and data visualization more natural, creating an environment that conveys valuable insights and clear actions related to general or specific aspects of the participating building ecosystem (virtualization). To cope with such aspects, we follow the concept of IoT as defined by ITU-T [10].

\section{Platform architecture, core services and implementation choices}

The overall platform architecture (Fig. 3) is built upon multiple heterogeneous installations in schools. The IoT devices continuously collect data from multiple sensing points and forward them to local gateways. Data messages retrieved from the end-devices are circulated within cloud services over a message bus system, responsible for distributing the information gathered to the subsystems responsible for data storage and processing. This allows us to introduce data processing, transformation, and aggregation mechanisms at different layers, depending on the needs of the high-end applications. As a result, the system scales more efficiently than a centralized solution. As GAIA's deployments expand, new IoT device types and communication technologies are added by creating adapters that convert device-specific messages to the internal data model of our IoT service platform and forwarding them to the internal message bus.

The message bus assigns a dedicated stream processing pipeline for each sensor supported by IoT devices. Sensor data streams are assigned unique names, derived from the unique network address of the sensor device producing the data, the type of sensor and the unique ID of the IoT device. The message bus layer relies on a Message Broker implementation, supporting both MQTT and AMQP protocols, by leveraging Mosquitto and RabbitMQ open-source software. MQTT is used by gateway devices to send measurements to our cloud infrastructure. The IPenabled IoT nodes (weather/atmospheric) use MQTT to publish data.

Data Analysis uses the Apache Storm distributed real-time computation system. For each processing type (e.g., average hourly consumption), we define a stream processing pipeline that processes a sensor data stream and extracts aggregates in real time. Sensors produce (periodically or asynchronously) events that are sent via RabbitMQ. Those events are usually 2-tuples, i.e., (value, timestamp) pairs. All data received are forwarded to a specific queue, where they are processed in real time by a Storm cluster. The Storm cluster has a number of topologies for processing based on the data type. Each topology is responsible for a unique type of environmental sensor (temperature, humidity, wind speed, etc.), actuators, power measurement sensors, etc. The produced analytics is outputted into summaries, which are stored permanently either to a file system or to a database in our storage module.

Information retrieval, transfer and manipulation in real-time allow small margins for erratic behavior. To deal with such issues, the processing pipeline uses three generic steps. The first identifies the presence of erroneous data that adversely affects the study of any time series. Such errors may be due to transient errors occurring on the device provider and should be excluded 
from the dataset. The second applies a 5-minute moving window to deal with non-uniformities and remove short-term fluctuations, by identifying outliers within the first and third quartile. The third step addresses uncertainties found on the data streams resulting from missing data. Missing data is a regular phenomenon in real-world IoT deployments, resulting from temporary disconnections of devices and entire networks due to limited device resources and the nature of lowpower wireless networks, or as result of end-users actions.

As soon as the 5-minute values are processed for each sensor stream, they are aggregated based on a variety of functions (e.g., $\mathrm{min} / \mathrm{max} / \mathrm{mean}$ ), and different intervals (e.g., 1-hour, 1-day). Firstorder and second-order analysis is applied to each stream to identify local maxima/minima and basic measures on the slope of the data. Four specific pipelines are defined to handle streams from simple sensors, actuators, power meters and weather data, and one more to generate notifications based on rules defined by the users and one topology to invoke automated actions like switching lights on/off, based on the measurements reported. A single virtual machine instance, running on an Intel E5-2630V4 processor with access to 6 cores and 24 GB RAM, can process up to 5833 messages per second.

Scalable Data storage is implemented at different layers of the architecture leveraging the message bus connection: (a) data are formatted for near-instantaneous retrieval, avoiding time-consuming queries and aggregations; (b) a cache-like mechanism is available for providing recent data for a specific sensor devices or collection of end-devices, using a single lookup in near-located storage service; (c) older historical measurements are stored in a deep storage service, used only when measurements older than the ones provided by the first service are needed (implemented using MongoDB). This decoupling of data generators and storage services allows us to implement a range of services that aim to optimize various performance criteria.

Sensor annotations and metadata are stored on a different storage than the actual sensor readings. This service stores the deployed sensors and their metadata (e.g., observed properties, measurement units) as part of a graph database, allowing complex relation queries like getting the list of available temperature sensors in multiple locations, by running a graph traversal query. Thus, the platform avoids the need for preconfiguring the IoT infrastructure and the fixed routing of data through the various cloud-based services that constitute the system. The implementation is based on neo $4 \mathrm{j}$ (neo4j.com), a high-performance graph database.

Accessing data is crucial for building monitoring applications, e.g., when comparing historical data from different time spans and building areas. Data Analysis service allows organizing large volumes of data and visualizing them from different points of view instantaneously, regardless of the targeted time interval. An open interface to query is provided for both stream-based and historical data by defining a list of sensor keys and time limits. The decoupling of data analysis, semantic annotation and storage services allows the implementation of a range of services that are required to optimize different performance criteria.

The Identity Management service provides user authentication and covers a number of aspects involving users' access to services and applications, including secure and private authentication from mobile devices and web applications, or user profile management and privacy-preserving disposition of personal data. All subsystems are protected by firewalls and authentication mechanisms. Access to various services is limited by different permission levels defined for user roles (students, teachers, building administrators).

Finally, developing applications on top of this architecture is simple, since the services described above can be accessed via well-defined APIs. The Data API comprises a Real-time Data API and a Historical Data API. The Real-time Data API is a streaming API, which gives low latency access to new data registered to the platform, while the Historical Data API allows retrieval of historical data registered by any device, as well as aggregated summaries (maximum, minimum, average). In addition, the Directory and Authentication/Authorization APIs allow to create and manage devices, users and authorization roles. Akrivopoulos et al. discuss such aspects in [11]. 


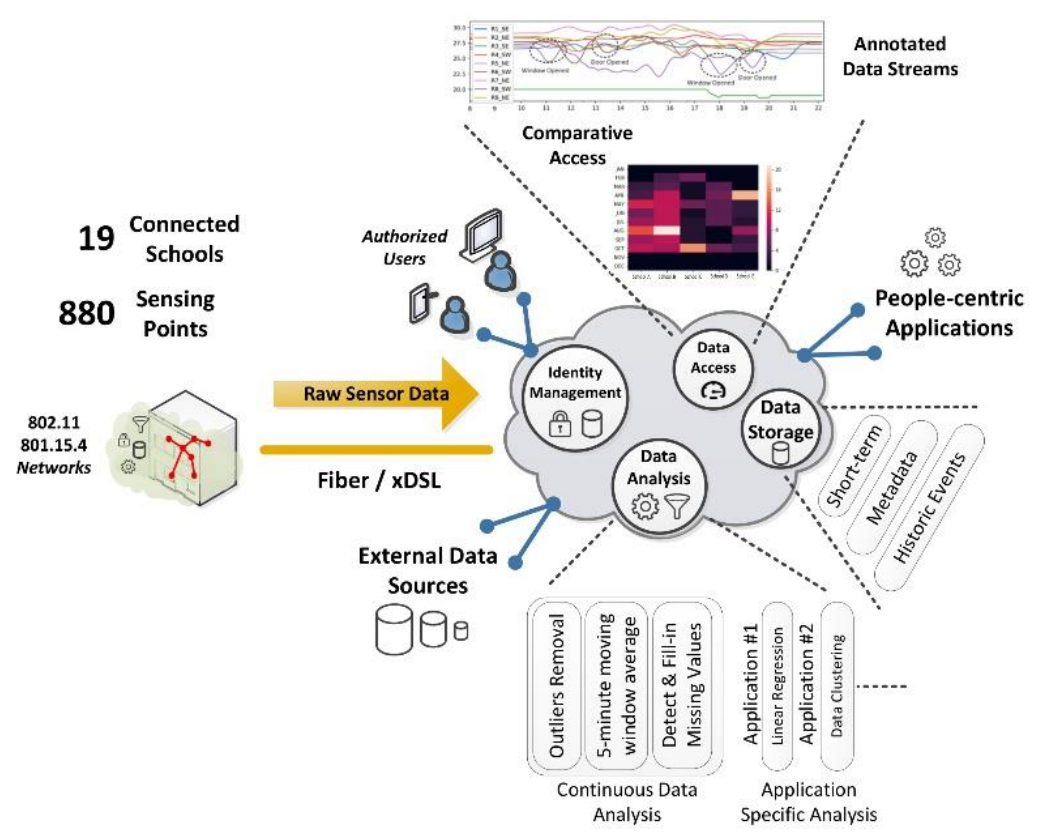

Fig. 3. Overall architecture of the system.

\section{EDUCATIONAL APPLICATIONS AND PRELIMINARY USER FEEDBACK}

To support IoT-enabled energy-related educational activities, while achieving behavioral change, our approach is focused around three pillars: raising awareness, supporting action, and fostering engagement. In this context, software and hardware artifacts have been produced interoperating with the GAIA IoT deployment: a) an online challenge (http://gaia-challenge.com), comprising gamification "quests" and activities aimed at students and classes, b) an online building management system providing users with near real-time feedback on energy consumption and environmental parameters, and c) templates for activities, called the Educational Lab kit, based on DIY hardware for students to assemble "installations" interacting with GAIA's platform. In addition, a recommendation engine proposes actions towards better efficiency based on the data gathered, addressed at building managers, teachers and students.

Including the users in the loop of monitoring their daily energy consumption is a first step towards raising awareness. In an educational environment, this step can be further enhanced in the context of educational activities through GAIA's application set. In essence, it complements an educational approach that encourages customization to the specific requirements of each school, which "fine-tune" the tools used, when and for how long, during a school year. Overall, the activities in each school are based on data produced within the respective buildings, while the effects of changing certain behaviors can be detected and quantified. E.g., teachers can extend existing educational activities on sustainability with simple actions with immediate IoT-enabled feedback, such as turning off the lights in parts of the building and monitoring the effects, or using thermal cameras to discover problematic areas combined with data showing the effects of lacking building insulation.

During 2017, we conducted preliminary tests over several weeks to get feedback regarding our implementation. Overall, 944 students and teachers had a first interaction with the GAIA software ecosystem, while we conducted a form-based survey focusing on the gamification component (196 high-school students in Sweden and Italy) and the Educational Lab Kit (132 $6^{\text {th }}$ graders in Greece). With respect to the game, $78 \%$ of the students found the content interesting (21\% "absolutely", 26\% "very", 31\% "moderately") and 89\% the activity user-friendly (38\% "absolutely", 29\% "very", 22\% "moderately"). Regarding the Lab Kit: 
- When asked "Would you repeat the same experiment in your home or inform your parents about it", students answered "Absolutely" 22\%, "Very much" 22\%, "Moderately" $21 \%$, "Slightly" $15 \%$ and "No" $20 \%$.

- To the question "Did it help you learn something about the building's behavior", students answered "Absolutely" 26\%, "Very much" 22\%, "Moderately" 29\%, "Slightly" $10 \%$ and "No" $13 \%$.

- When asked "Would you like to repeat such an activity", 56\% answered "Very much" and "Absolutely", 16\% "Moderately" and 28\% "Slightly" and "No".

- $45 \%$ answered they had previous experience with electronics.

For school year 2017-18, we refined and expanded lab activities significantly. We had additional evaluation rounds, with a total of 48 and 58 students aged 11-15 participating respectively in 2 labs, without previous participation. In the first lab, $71 \%$ answered that they absolutely liked the activity (5/5), $23 \%$ gave out $4 / 5$, and the rest $3 / 5$. In the second lab, there was some change to the positive response, with $58 \%$ rating it $5 / 5,26 \% 4 / 5$, and $12 \% 3 / 5$, possibly also reflecting the change of topic for the lab. A question was included for students, whether they "found any difficulty in implementing the lab activities"; $80 \%$ stated that they did not face any difficulties. From our direct interaction with teachers and students, we noticed that teachers prefer a minimal, structured experience that enables them to use the data inside a 45-minute lecture.

In order to investigate overall learning performance outcomes regarding students, we produced questionnaires and had interviews with 5 teachers from 5 different schools, whose classes participated in the lab activities, over a period of 4 months belonging to the abovementioned student group. To the question "have you noticed any substantial change with respect to students' performance after GAIA activities began?" 1 teacher noticed a "mild change", while the 4 others answered with "very significant change". In free text answers, they noted "positive changes in daily class activity and greater interest towards programming" and that "students who previously were indifferent, now have significant participation during lab activities". Another teacher noted that "low performing students had a chance to exhibit their capabilities and receive positive comments from the rest of their class".

Regarding other learning enhancements in the schools involved, GAIA has enabled additional capabilities in some of the schools, as well as provided options that did not exist previously in others. E.g., in Sweden, technical college students constructed and installed the nodes themselves as part of their classes, helping them to boost their knowledge in related technologies. In an Italian technical high school, some classes have achieved very good scores in the gamification part of GAIA. They have also produced content utilizing sensor data from their school building and are actively monitoring lighting with simple actions, achieving in certain places reductions of up to $17 \%$, when comparing May 2017-2018.

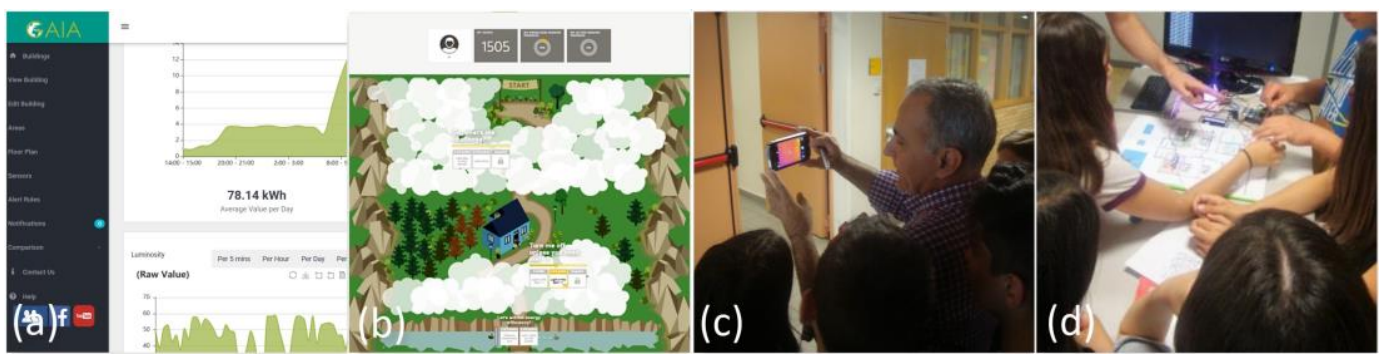

Fig. 4. (a) A screenshot from the BMS application, (b) the board game-like UI of GAIA Challenge, (c) educational activity using thermal cameras "mapping" a school building energy profile, and (d) students using DIY electronics to interface with GAIA and visualize energy data. 


\section{DATA-DRIVEN LESSONS LEARNED}

Ensuring the energy-efficient and sustainable operation of school buildings is an extremely complex process, since each building has vastly different characteristics in terms of size, age, location, construction, thermal behavior and users. IoT platforms can provide quantitative evidence to evaluate and improve organizational and managerial measures. The business value and benefits of analyzing the data collected from our IoT platform are presented here.

The IoT platform enables direct comparisons of energy efficiency between buildings, carefully taking into account many environmental parameters. The comparative evaluation of the energy consumption of schools identified a certain school with unusually high expenditure. This school building housed a public school and a private technical school, operating at different times of the day. This is common in Greece, where schools can share facilities with other entities. Data analysis indicated that one school consumed significantly more electricity than the other, as well as that a private entity renting part of the building consumed more electricity than the rent paid.

In another school building located in an area with harsh conditions during winter months, the observed temperature data show that at least one day is required each week after the weekend to reach comfortable conditions inside the building, i.e., the temperature drops below $15^{\circ} \mathrm{C}$ during the weekend and a full day of heating is needed to return to $18^{\circ} \mathrm{C}$ (i.e., on Mondays the building is cold). Such data can be utilized by building managers to predict the average time that buildings need to be heated or cooled during wintertime or summertime, effectively lowering the buildings' energy footprint.

Examining the classrooms' temperature helps to understand the conditions under which students and teachers operate. Several factors affect classroom temperatures, ranging from the local weather conditions to the room's orientation, construction materials, insulation, and radiators' placement. From our analysis of indoor and outdoor (temperature, precipitation) data, we verified that temperatures can go quite high, with indoor temperatures exceeding outdoor ones more than $10^{\circ} \mathrm{C}$. We also observed (Fig. 5a) that classrooms facing southwest are exposed for longer periods, thus maintaining higher temperatures for longer periods than classrooms with a different orientation. Employing such data mining techniques over real-world IoT data can provide insights to managers/staff about possible interventions for improving indoor conditions, depending on the schools' physical characteristics.

Another factor affecting temperature inside classrooms is the daily activity of students and teachers. Additionally, the continuous operation of the IoT platform allows the collection of data during periods with no such activity. Comparing indoor conditions of classrooms with and without activity indicated that opening and closing doors/windows/blinds has an immediate effect on temperature, dropping it by $2^{\circ} \mathrm{C}$. Given these considerations, we analyzed temperature data to identify poorly performing classrooms. Fig. $5 \mathrm{~b}$ shows findings from two schools in the same city. Observing the data, we can identify some specific problems. In the bottom graph, we see that room B1 has poor performance, with a temperature increase from $20^{\circ} \mathrm{C}$ to $32^{\circ} \mathrm{C}$ in the span of 8 hours. In the top graph, we observe that classrooms facing SW (A4) and SE (A4) have an increase of $2^{\circ} \mathrm{C}$, whereas rooms in the bottom school, even those with the same orientation, are unaffected. Such results can provide useful insights to building managers about ways to improve classroom conditions by arrangement of indoor spaces, strategic landscaping, and modifications to the building envelope.

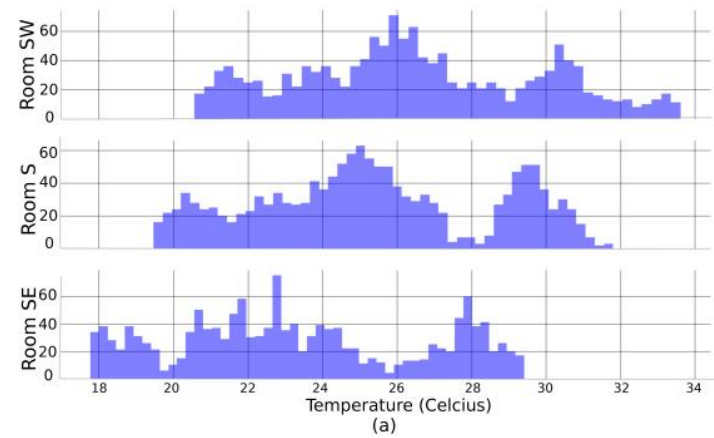

(a)

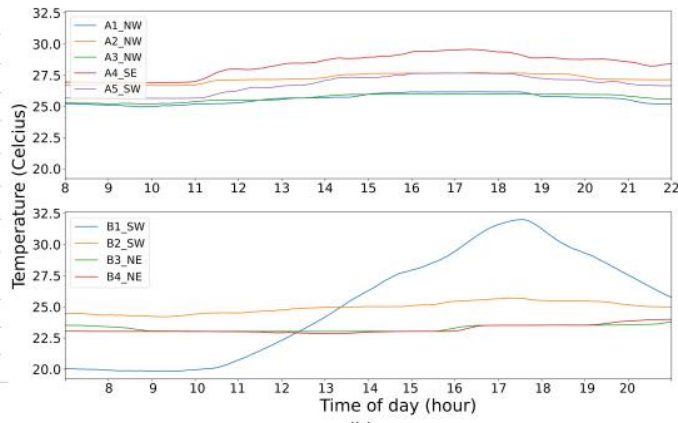

(b) 
Fig. 5. Analysis of building performance: (a) indoor temperature histogram for three classrooms with different orientation (SW, S, SE) during Sep/17 to Oct/17, (b) comparison of temperatures in classrooms of two schools on 30/Sep/17.

The examples above focused on power consumption and indoor/outdoor temperature. Given the variety of sensors deployed inside school buildings, a data mining approach can be applied to investigate conditions within classrooms: lighting, air quality and noise are also critical parameters. We now give an example of energy savings potential related to lighting that came out by examining a day's consumption in cooperation with a group of one teacher and some students in a specific school building in Italy. In that school, lights in the central hall of the building at the start of the day required 1.9KW, while from 10:00 - 17:00 all lights were on, requiring 4.9KW. Examining the indoor luminosity data, sensors indicated that this was unnecessary, since illuminance from natural light was close to 400 lux (good indoor lighting conditions). This was a recurring scenario in this building. With a conservative estimate of 60 sunny days a year we have $1260 \mathrm{KWh}$ in savings.

\section{CONCLUSIONS}

We discussed an education-focused real-world deployment in schools in Europe, aimed at promoting sustainability and energy awareness. By using this infrastructure and the data it produces, it is easier and more effective to build tools that better reflect the everyday reality in school buildings and provide a more meaningful feedback. Our platform has allowed the development of educational activities aiming to link real-world data with sustainability classes in schools participating in the GAIA project. Our pilot study across several school buildings so far provided positive feedback and useful insights on how such a platform can be utilized to promote sustainability awareness and behavioral change towards energy savings. Additional long-term educational activities based on this platform will be conducted, while we also plan to extend GAIA's infrastructure to additional school buildings.

\section{ACKNOWLEDGMENTS}

This work has been supported by the EU research project "Green Awareness In Action", funded under H2020 and contract number 696029.

\section{REFERENCES}

1. "Key data on education in Europe 2012", Education, Audiovisual and Culture Executive Agency (EACEA P9 Eurydice, EUROSTAT), 2012.

2. Nicolae, L. (2005) Council of Europe Plenary Session: Youth Education for Sustainable Development.

3. "Europe's buildings under the microscope: A country-by-country review of the energy performance of buildings," Buildings Performance Institute Europe, 2011.

4. Crosby, K.; Metzger, A.B., "Powering down: A toolkit for behavior based energy conservation in K-12 schools," U.S. Green Building Council, 2012.

5. Froehlich, J.; Findlater, L.; Landay, J., "The design of eco-feedback technology," in CHI '10. New York, NY, USA: ACM, 2010, pp. 1999-2008.

6. Alvarez-Campana M, López G, Vázquez E, Villagrá VA, Berrocal J. Smart CEI Moncloa: An IoT-based Platform for People Flow and Environmental Monitoring on a Smart University Campus. Sensors. 2017;17(12):2856.

7. Sustainable Energy Across the Common Space, http://en.seacs.eu/

8. Pocero, L.; Amaxilatis, D.; Mylonas, G.; Chatzigiannakis, I., Open Source IoT Meter Devices for Smart and Energy-Efficient School Buildings, in: HardwareX, volume 1, 54-67, 2017. 
9. Shelby, Z.; Hartke, K.; Bormann, C., "The Constrained Application Protocol (CoAP)," RFC 7252, IETF, 2014.

10. ITU-T Y.4000, "Overview of the Internet of Things", Telecommunication Standardization Sector of ITU, 2016.

11. Amaxilatis, D.; Akrivopoulos, O.; Mylonas, G.; Chatzigiannakis, I. An IoT-Based Solution for Monitoring a Fleet of Educational Buildings Focusing on Energy Efficiency. Sensors 2017, 17, 2296.

\section{ABOUT THE AUTHORS}

Georgios Mylonas is a researcher at Computer Technology Institute and Press "Diophantus", Patras, Greece. His research interests include IoT, distributed and pervasive systems. He received his $\mathrm{PhD}$ in Computer Science from the University of Patras, and is the coordinator of the GAIA project. Contact him at mylonasg@cti.gr.

Ioannis Chatzigiannakis is an Associate Professor at the Department of Computer, Control \& Informatics Engineering, Sapienza University, Italy. He received a PhD in Computer Science from the University of Patras. His research interests include IoT, Pervasive and Distributed Computing, Algorithmic and Software Engineering. Contact him at ichatz@diag.uniroma1.it.

Dimitrios Amaxilatis is a researcher at the Computer Technology Institute in Patras, Greece. He currently pursues his PhD at the University of Patras. His research interests include distributed algorithms, smart cities and participatory sensing. Contact him at amaxilat@cti.gr.

Federica Paganelli is a senior Researcher at Consorzio Nazionale Interuniversitario per le Telecomunicazioni, Florence, Italy. She received a PhD in Telematics and Information Society from the University of Florence. Her research interests include service composition, network virtualization, software-defined infrastructures, context-aware systems and IoT. Contact her at federica.paganelli@unifi.it.

Aris Anagnostopoulos is an Associate Professor at the Department of Computer, Control, and Management Engineering at Sapienza University, Italy. He received a PhD in Computer Science from Brown University. Before Sapienza, he was with Yahoo! Research, USA. Contact him at aris@dis.uniroma1.it. 